\title{
AN IMPROVED MODEL FOR THE PENETRATION OF A RIGID PROJECTILE INTO A DUCTILE TARGET
}

\author{
S. SYNGELLAKIS \\ Wessex Institute of Technology, U.K.
}

\begin{abstract}
A simple penetration model for rigid projectiles against ductile targets is enriched by the introduction of an exponential parameter that extends the validity of the model to projectiles with hemispherical heads. The application of the analysis to various head cases is demonstrated with emphasis on the hemispherical head for which simpler relations are derived and the equation of motion explicitly integrated. Resort to the considerable amount of numerical and experimental penetration data available in the literature allows the rational estimation of the various parameters that characterise the model.

Keywords: characterisation, ductile target, hemispherical head, modelling, ogival head, penetration, rigid projectile.
\end{abstract}

\section{INTRODUCTION}

The phenomena arising from high-energy impact on thick plates vary considerably with the type, range and intensity of attack, as well as plate geometry, material and support conditions. With the trend towards more penetration-resisting plating, more of the impact energy would be transmitted to and absorbed by the rest of the structure. This development has important implications on the design of the plate supports. Moreover, by studying the shock wave attenuation through the structure, it would be possible to assess the intensity of the excitation at selected points and hence the effect of the shock on sensitive equipment.

A rational design of structural elements against impact loads relies on a good approximation of the force arising during a missile-target interaction. Computational techniques are nowadays extensively used to model this complex interaction and predict its consequences. However, in problems where greater interest is placed on the overall response of the structural element and its connections with the whole structure, more easily tractable models for the impact force are desirable. A recent review article discussed the basic assumptions of such modelling and tested its effectiveness by referring to available, numerically or experimentally obtained, penetration data [1].

For given configurations and material characteristics of a projectile and its target, the projectile velocity is the most fundamental factor having a profound effect on the impact phenomenon [2]. For a certain, experimentally determined velocity limit, the projectile penetrates into an elasto-plastically deformed target without itself sustaining any significant deformation. In such cases, the impact phenomenon is modelled as a penetration of a rigid projectile into a ductile target.

The impact force is equal to the resistive force applied by the target on the projectile during the latter's penetration. Its time history can be obtained by solving the equation governing the motion of a rigid projectile:

$$
m \ddot{x}=-F(x, \dot{x})
$$

where $F$ is the resistive force, $x$ the projectile penetration, $m$ the projectile mass and a dot above a symbol indicates differentiation of the represented variable with respect to time. As implied by eqn (1), $F$ would generally depend on both penetration and projectile velocity. 
If the projectile is assumed axisymmetric and its head shape is described in cylindrical coordinates by a function $r=r(z)$, as shown in Fig. 1 , then $F$ is given by

$$
F=2 \pi \int_{0}^{r_{e}} p(x, \dot{x}) r \mathrm{~d} r
$$

where $p$ is normal pressure over the projectile-target interface and $r_{e}$ the radius of the edge of the contact area. In Fig. 1, the projectile head of length $L_{h}$ merges with the shank of calibre $d$ at bourrelet.

Simple models for pressure $p$ found in the literature usually comprise two terms: the first is a hardness term essentially depending on the rate-dependant mechanical properties of the target material, the second can be referred to as hydrodynamic since it is related to the kinetic energy of the plastically flowing target material around the penetrating projectile.

In the initial penetration phase, $r_{e}=r_{x}=r\left(z=L_{h}-x\right)$ as illustrated in Fig. 2a. When the impact velocity is very low, maximum $x$ could be less than $L_{h}$; in such a case, the quasi-static part of the pressure predominates and the hydrodynamic part can be neglected; thus, according to eqn (2), the resisting force depends mainly on penetration. For moderately high-impact velocities, $r_{e}$ reaches $d / 2$, its maximum value, as shown in Fig. 2b. Above a certain impact velocity, the phenomenon of cavitation occurs [3] and contact between the projectile head and target is lost for $r_{e}=r_{c}$ less than $d / 2$, as shown in Fig. 2c. In this penetration mode, the role of the hydrodynamic pressure term becomes important.

To obtain an expression for the impact force from eqn (2) and thus enable the integration of eqn (1), a plausible model for pressure $p$ needs to be adopted. This can be achieved by

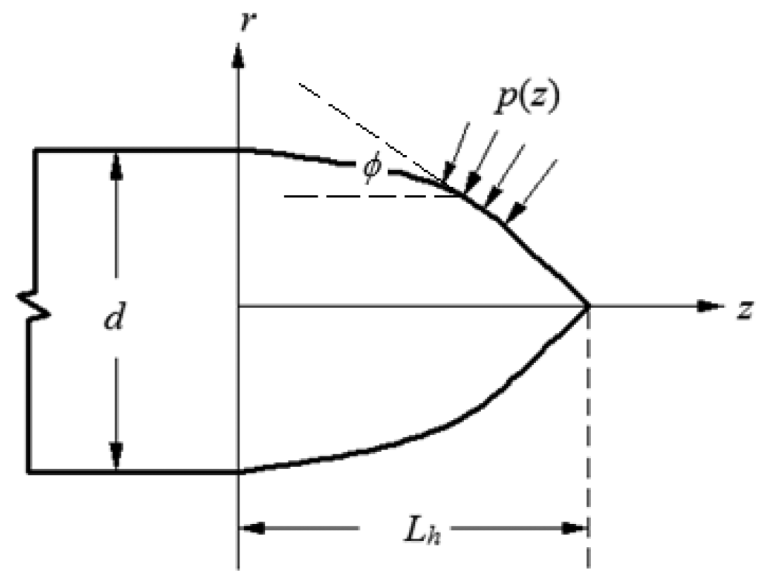

Figure 1: Projectile head profile subjected to normal pressure $p$.

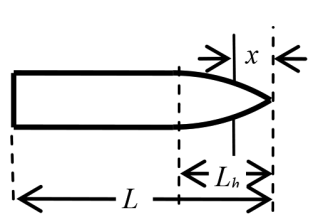

(a)

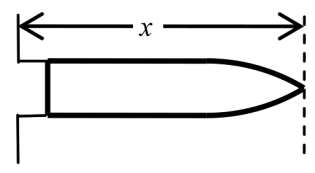

(b)

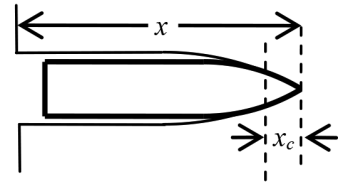

(c)

Figure 2: The three main penetration modes. 
resorting to one such simple model among the various that have been proposed and can be found in the literature [3-8].

The focus of the present article is a particular pressure model proposed by Hill [3], which accounts for the influence of the head shape; although this model works well for ogival and conical heads, it was not found to be altogether satisfactory in the case of hemispherical heads for which it fails to predict a smooth transition from no cavitation to cavitation behaviour.

An attempt is here made to generalise Hill's model so that it accounts properly for hemispherical heads. A rational modification is introduced allowing the consistent application of the model to all types of head shapes. The characterisation of the head shape effect is achieved through the fitting of model predictions to experimental measurements.

\section{BACKGROUND}

\subsection{Projectile head geometries}

The head profile in the shape of a circular arc of radius $n d$, where $n$, a constant parameter greater than $1 / 2$, is known as the calibre-radius-head (c.r.h) or the ogive. An ogival head is generated by the rotation of the ark about the $z$ axis; it is pointed and merges smoothly with the cylindrical base. In polar coordinates (Fig. 1), its profile is represented by the equation,

$$
r(z)=\left(n^{2} d^{2}-z^{2}\right)^{1 / 2}-(n-1 / 2) d
$$

It is easily shown that the ogive head length is given by

$$
L_{h}=z(r=0)=(n-1 / 4)^{1 / 2} d
$$

Alternatively, the profile can be described parametrically by

$$
r(\phi)=n d \cos \phi-(n-1 / 2) d, z(\phi)=n d \sin \phi,
$$

where $\phi$ is the angle between the tangent to the profile and the $z$ axis (Fig. 1) so that $(\mathrm{d} r / \mathrm{d} z)=$ $-\tan \phi$. At the tip, $f_{0}=\phi\left(0, L_{h}\right)$ is given by

$$
\tan \phi_{0}=\frac{(n-1 / 4)^{1 / 2}}{n-1 / 2}
$$

which means that head slenderness $\left(\phi_{0} \rightarrow 0\right)$ corresponds to $n \rightarrow \infty$.

When $n=1 / 2$, the head shape becomes hemispherical, which also merges smoothly with the cylindrical base but is not pointed. In contrast, the conical head, for which $\phi$ is constant, is pointed but does not merge smoothly with the cylindrical base; its profile is described by

$$
r(z)=\left(L_{h}-z\right) \tan \phi
$$

Finally, it is possible to form a composite head by grinding a conical tip on an ogive. The geometry of this head is fully defined by the ogive parameter $n$, as well as the vertex angle $2 \phi_{t}$ and the basal radius $r_{t}$ of the conical cap. The penetration analysis for composite heads is, as expected, more complicated than that for plain ones but Hill facilitated it by postulating a simple rule governing the calculation of the resisting force $F[3]$. 


\subsection{Pressure formula}

In most simplified penetration analyses [3-8], the normal pressure on the projectile head is represented by the expression

$$
p(z, \dot{x})=p_{0}+b(z) \rho \dot{x}^{2}
$$

where $p_{0}$ is the hardness term, $b(z)$ plays the role of a shape drag coefficient [1] and $\rho$ is the density of the target material. Assuming the response of the target material to be elasto-plastic, the quasi-static part of the pressure can be expressed as

$$
p_{0}=a \sigma_{Y}
$$

where $\alpha$ is a constraint factor and $\sigma_{Y}$ the strain rate-sensitive yield or flow stress.

Hill neglected the frictional component of interface traction because of surface melting and adopted eqn (8) for the normal component with $b(z)$ given by

$$
b(z)=K \frac{\mathrm{d}}{\mathrm{d} z}\left(r \frac{\mathrm{d} r}{\mathrm{~d} z}\right)
$$

where $K$ is a dimensionless, positive parameter, found experimentally to depend on head shape and target strength. It is easily shown that, in the case of conical head,

$$
b(\phi)=K(\phi) \tan ^{2} \phi
$$

The inspiration for eqn (8) with $b$ given by eqn (10) was a pressure formula obtained for very slender heads of any shape assuming cylindrical expansion of the elasto-plastic target material around the penetrating projectile [3]. According to this formula, for a slender conical head,

$$
K(\phi \rightarrow 0)=\ln \left(\frac{G}{\tau_{Y}}\right)^{1 / 2}
$$

where $G$ is the modulus of rigidity and $\tau_{Y}$ the shear yield stress of the target material. For slender ogival heads, eqn (11) provides an estimate of $K(n \rightarrow \infty)=K\left(\phi_{0} \rightarrow 0\right)$, according to eqn (6).

\subsection{Cavitation velocity}

The critical condition marking the beginning of cavitation develops for a critical velocity $\dot{x}_{c}$ when pressure $p$ vanishes at the bourrelet, that is, for $z=0$. Using eqn (8) with $b$ given by eqn (10), it is possible to show that, for ogival and hemispherical heads, $\dot{x}_{c}$ is given by

$$
\dot{x}_{c}=\left(\frac{2 n p_{0}}{K \rho}\right)^{1 / 2}
$$

\section{A NEW HYDRODYNAMIC PRESSURE MODEL}

It was pointed out in Section 2.2 that Hill was inspired into adopting eqn (10) for the drag coefficient by assuming a cylindrical expansion of the target material around the penetrating 
projectile. This is a reasonable assumption for a slender, pointed projectile such as one with an ogival or a conical head. A blunt spherical head however would be expected to produce, at least initially, a spherical expansion of the target material. This consideration led to the modified modelling of the drag coefficient described in this section.

Hill's model for the hydrodynamic pressure distribution over the projectile head is here generalised by adopting for the drag coefficient the formula

$$
\begin{gathered}
b(z)=K\left(\frac{4 r}{d^{2}}\right)^{k-1} \frac{\mathrm{d}}{\mathrm{d} z}\left(r^{k} \frac{\mathrm{d} r}{\mathrm{~d} z}\right) \\
\text { or } \\
b(z)=K\left(\frac{2 r}{d}\right)^{2 k-2}\left[k\left(\frac{\mathrm{d} r}{\mathrm{~d} z}\right)^{2}+r \frac{\mathrm{d}^{2} r}{\mathrm{~d} z^{2}}\right]
\end{gathered}
$$

where the positive, not necessarily integer exponent $k$, is left for the moment unspecified; a plausible value for $k$ will be deduced later through the application of the model to specific head shape cases. It is clear that, for $k=1$, eqn (13) coincides with Hill's formula, eqn (10). Substituting eqn (8) with $b$ given by eqn (13) into eqn (2) and integrating leads to the expression for the resistive force

$$
F=\pi r_{e}^{2}\left[p_{0}+K \rho \dot{x}^{2}\left(\frac{2 r_{e}}{d}\right)^{2 k-2} \tan ^{2} \phi_{e}\right]
$$

As mentioned in the Introduction, for moderately high-impact velocities, the whole head eventually comes into contact with the target. Provided that the head merges smoothly with the cylindrical base, $\phi_{e}=\phi(1 / 2 d, 0)=0$ and the resistive force reduces to

$$
F=A p_{0}
$$

where $A=1 / 4 \pi d^{2}$ is the cross-sectional area of the projectile base. Thus, during this second stage of penetration, the resistive force is constant depending only on the quasi-static part of the dynamic pressure.

Application of the cavitation condition, $p(1 / 2 d, 0)=0$, provides the critical velocity above which cavitation occurs so that the hydrodynamic pressure also contributes to the resistive force. Using eqn (8), with $b$ given by eqn (14), and assuming again that the head merges smoothly with the cylinder at the bourrelet, the cavitation condition leads to

$$
p_{0}+K \frac{d}{2}\left[\frac{\mathrm{d}^{2} r}{\mathrm{~d} z^{2}}\right]_{z=0} \rho \dot{x}_{c}^{2}=0
$$

Eqn (17) is independent of $k$; it should therefore result in the same cavitation velocity as that predicted by Hill for $k=1$.

\subsection{Ogival head}

The analysis is facilitated by the introduction of the dimensionless coordinate $\zeta=z / d<n$. In terms of $\zeta$, the profile equation (3) and the derivatives of $r(z)$ are written 


$$
\begin{gathered}
r(\zeta)=\left[\left(n^{2}-z^{2}\right)^{1 / 2}-(n-1 / 2)\right] d \\
\frac{\mathrm{d} r}{\mathrm{~d} z}=-\frac{\zeta}{\left(n^{2}-\zeta^{2}\right)^{1 / 2}}, \frac{\mathrm{d}^{2} r}{\mathrm{~d} z^{2}}=-\frac{n^{2}}{\left(n^{2}-\zeta^{2}\right)^{3 / 2} d}
\end{gathered}
$$

It is thus confirmed that the cavitation velocity obtained from eqn (17) would be given again by eqn (12), a result, as expected, independent of $k$. Substituting eqn (19) into eqn (14), the formula for the drag coefficient in the hydrodynamic pressure distribution becomes

$$
b(\zeta)=K\left(\frac{2 r}{d}\right)^{2 k-2}\left[\frac{k \zeta^{2}}{n^{2}-\zeta^{2}}-\frac{r}{d} \frac{n^{2}}{\left(n^{2}-\zeta^{2}\right)^{3 / 2}}\right]
$$

As pointed out in the previous section, for impact velocities lower than $\dot{x}_{c}$, the impact force is given by eqn (16). In the presence of cavitation $\left(\dot{x}>\dot{x}_{c}\right)$, contact is lost away from the bourrelet, at $r_{e}=r_{c}<1 / 2 d, z_{e}=z_{c}>0$ and $\phi_{e}=\phi_{c} \neq 0$; then, the resisting force is determined from eqn (15) after obtaining the corresponding value of $z_{c}$ from the condition

$$
p\left(\zeta_{c}\right)=p_{0}+K \rho \dot{x}^{2}\left(\frac{2 r_{c}}{d}\right)^{2 k-2}\left[\frac{k \zeta_{c}^{2}}{n^{2}-\zeta_{c}^{2}}-\frac{r_{c}}{d} \frac{n^{2}}{\left(n^{2}-\zeta_{c}^{2}\right)^{3 / 2}}\right]=0
$$

Taking into account eqns (12) and (18), the above equation can be reformulated as

$$
\psi^{3}+n \alpha^{2}[2 \psi-(2 n-1)]^{2 k-2}\left\{2 \psi\left[(k-1) n^{2}-k \psi^{2}\right]+n^{2}(2 n-1)\right\}=0
$$

where

$$
\psi=\left(n^{2}-\zeta_{c}^{2}\right)^{1 / 2}, \alpha=\frac{\dot{x}}{\dot{x}_{c}}
$$

Considering that $\alpha>1$ and $0<\zeta_{c}<(n-1 / 4)^{1 / 2}$, it is possible to show that $n-1 / 2<\psi<n$; therefore, if a solution of eqn (22) exists, it lies within a rather narrow range and can be easily obtained by some numerical technique such as the Newton-Raphson method. Then $\zeta_{c}$ and $r_{c}$ are calculated using the first of eqns (23) and (18), respectively. Substituting these values into eqn (15), the force on the projectile can be obtained in the form

$$
F=\lambda^{2} A p_{0}
$$

where $\lambda$ is the ratio of cavity to projectile diameter given by

or, in terms of $\psi$,

$$
\lambda=\frac{2 r_{c}}{d}\left[1+2 n \alpha^{2}\left(\frac{2 r_{c}}{d}\right)^{2 k-2} \frac{\zeta_{c}^{2}}{n^{2}-\zeta_{c}^{2}}\right]^{1 / 2}
$$

$$
\lambda=[2 \psi-(2 n-1)]\left\{1+2 n \alpha^{2}[2 \psi-(2 n-1)]^{2 k-2}\left(\frac{n^{2}}{\psi^{2}}-1\right)\right\}^{1 / 2}
$$

It is apparent from eqn (26) that $\lambda$ depends on $a, n$ and $k$. For $k=1$, an explicit form is obtained for $\lambda$ as follows [1] 


$$
\lambda^{2 / 3}=(2 n)^{2 / 3}\left(2 n \alpha^{2}\right)^{1 / 3}-(2 n-1)^{2 / 3}\left(2 n \alpha^{2}-1\right)^{1 / 3}
$$

which was correlated to experimental evidence to yield estimates for the parameters $p_{0}$ and $K$ for particular values of $n$.

\subsection{Hemispherical head}

The hemispherical head can be considered as a special case of the ogival if $n$ is set equal to $1 / 2$. Then

$$
r(\zeta)=\left(1 / 4-z^{2}\right)^{1 / 2} d
$$

and

$$
b(\zeta)=2^{2 k-2} K\left(1 / 4-\zeta^{2}\right)^{k-2}\left(k \zeta^{2}-1 / 4\right)
$$

The above relation indicates that for the pressure distribution function to be finite at $\zeta=L_{h} / d$ $=1 / 2, k$ should be at least 2 . Adopting the value $k=2$,

$$
b(\zeta)=4 K\left(2 \zeta^{2}-1 / 4\right)=K\left(2 \sin ^{2} \phi-1\right)
$$

The value $k=2$ for the exponent in eqn (13) is consistent with the three-dimensional motion associated with spherical cavity expansion, which is the mode of deformation expected to be produced by a spherical head at the front of the propagating cavity.

The pressure distribution with $b$ given by eqn (30) is not uniform and the application of the cavitation condition mentioned in Section 2.3 leads to a cavitation velocity also given by eqn (12) with $n=1 / 2$. Substituting $n=1 / 2$ and $k=2$ into eqn (22) and solving the latter equation for $\zeta_{c}$ gives

$$
\zeta_{c}=(1 / 2 \sqrt{2})\left(1-1 / \alpha^{2}\right)^{1 / 2}
$$

Thus, according to the new model, contact between head and the target decreases smoothly as the penetration velocity increases from $\dot{x}=\dot{x}_{c}(\alpha=1)$ reaching a minimum corresponding to $z_{c}=d / 8^{1 / 2}$ for $a \rightarrow \infty$. In contrast, adopting $k=1$ according to Hill's model leads to uniform head pressure for $a<1$ and contact only at the tip with infinite pressure there for $a \geq 1$ [3]. From either eqn (25) or eqn (26), the ratio of cavity to projectile diameter is obtained as

$$
\lambda=\frac{1+\alpha^{2}}{2 \alpha}
$$

\subsection{Conical head}

The profile of a conical head is described by the equation

$$
r(z)=\left(L_{h}-z\right) \tan \phi=1 / 2 d-z \tan \phi
$$

where the gradient angle $\phi$ is constant in this case. The pressure distribution is now given by

$$
p(z)=p_{0}+K \rho \dot{x}^{2}\left(\frac{2 r}{d}\right)^{2 k-2} k \tan ^{2} \phi
$$


it is, therefore, non-uniform for $k>1$ but cannot vanish for any velocity, thus cavitation occurs only at the bourrelet. The resistive force is obtained from eqn (15) as

$$
F(x, \dot{x})=r_{e}^{2}\left[p_{0}+K \rho \dot{x}^{2}\left(\frac{2 r_{e}}{d}\right)^{2 k-2} \tan ^{2} \phi\right],
$$

Thus, $F$ depends on both penetration and projectile velocity only during the initial penetration phase, that is, for $r_{e}=x \tan \phi<1 / 2 d$; when $r_{e}=1 / 2 d$, it becomes independent of $k$ and projectile motion is analysed as described by Hill [3] who used the drag model represented by eqn (10).

\section{RESULTS AND DISCUSSION}

The proposed model makes the distinction between a pointed, ogive, and a blunt, i.e. hemispherical, projectile head. The former is characterised by a large $n(n \rightarrow \infty)$ and can be associated with a cylindrical expansion of the target material around the penetrating projectile; $k=1$ is therefore a reasonable assumption in this case. In the case of hemispherical head $(n=1 / 2)$, a spherical cavity expansion seems more plausible and therefore the value $k=2$ more appropriate. The relation between a normalised cavitation cross-section area and impact velocity for these two extreme cases is depicted in Fig. 3. The curve for $n=1 / 2, k=2$ represents eqn (31) while the relation for $n=\infty, k=1$ is deduced from eqn (27).

It is worth noting the proximity of the two curves in Fig. 3. The graphical representation of the same relation for any $n$ is expected to lie between the two extreme cases and it can be obtained if $k$ could be linked analytically with $n$. It is reasonable to assume that $k$ drops quickly from 2 to 1 as $n$ increases from $1 / 2$ to higher values encountered in experimental investigations involving ogival heads. For instance, a relation of the form $k=\mathrm{e}^{-2 n+1}+1$ would reflect such a quick transition since it gives $k=1.007$ for $n=3$, a value characterising the ogival nose projectiles used by Forrestal and co-workers in their ballistic experiments [4-8].

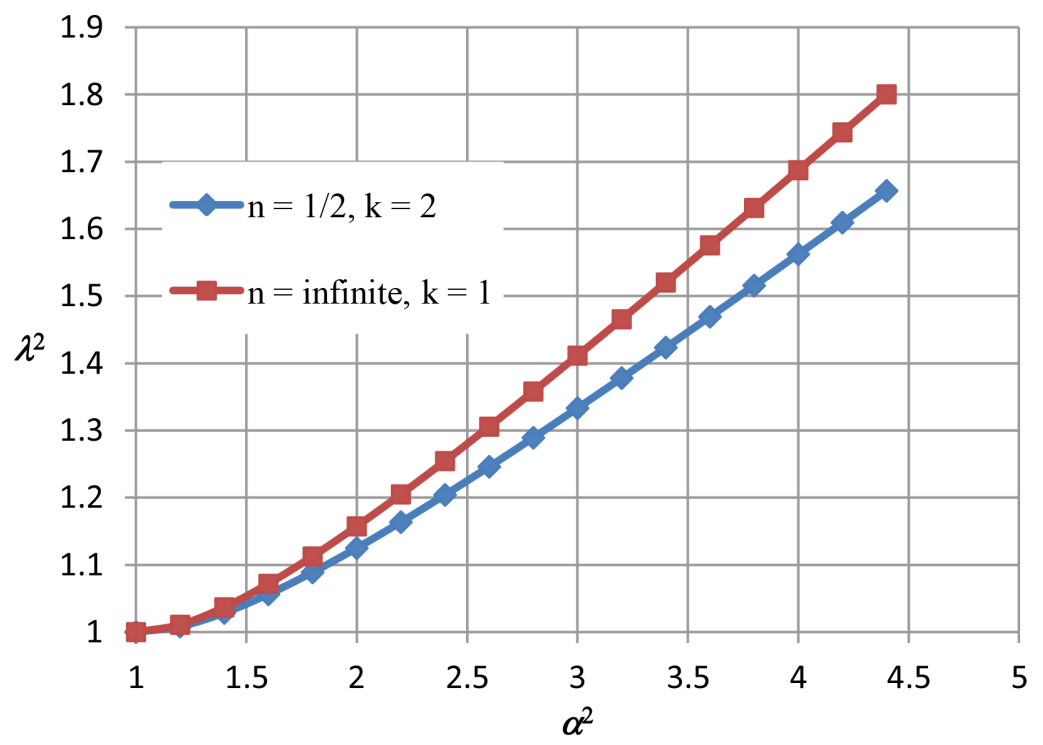

Figure 3: Normalised cavitation cross-section area versus normalised impact velocity. 
The empirical parameters of the model are determined by comparing its predictions with those of numerical simulations or with experimental measurements. Hill attempted this but relied on a rather limited amount of penetration data [3]. Since then, a significant numerical and experimental output has appeared in the technical literature.

Starting with hemispherical heads for which the analysis is much simpler, reference can first be made for comparisons to relevant finite element modelling (FEM) work [9, 10]. A key output from the analysis of Batra and Wright, who assumed a rigid-perfectly plastic target [9], was a set of pressure profiles for a range of penetration velocities. It is worth noting from these results that the pressure was found independent of velocity at approximately $\phi=45^{\circ}$, which is perfectly consistent with eqn (30). This result was subsequently confirmed by an FEM analysis, which accounted for the elasticity of the target [10].

The pressure profile predictions of Batra and Wright [9] allowed the estimation of parameter $a$ in eqn (9) at approximately 4.06, which is consistent with values suggested in the literature [11]. Since cavitation was not anticipated by FEM, positive pressure was predicted over part of the head above a certain velocity, which can be considered the cavitation velocity. For a velocity equal to $\left(0.72 \sigma_{Y} / \rho\right)^{1 / 2}$, the pressure was predicted positive over the whole head; using the extreme values of this pressure profile resulted in $K=3.41$. With this value of $K$, the cavitation velocity was predicted equal to $\left(1.19 \sigma_{Y} / \rho\right)^{1 / 2}$.

Comparisons were also made with the experimental results of Forrestal and co-workers using long rods with hemispherical heads [4]. The effect of the initial penetration phase on the overall penetration was considered small and it was thus neglected. Assuming first no cavitation, the maximum penetration obtained from eqn (1) with $F$ given by eqn (16) is

$$
x_{\max }=\frac{m \dot{x}_{0}^{2}}{2 A p_{0}}
$$

The above formula was applied using the data provided by Forrestal et al., $(d=7.1 \mathrm{~mm}$, $\rho=2,710 \mathrm{~kg} / \mathrm{m}^{3}$ ). In the first set of experiments [4], there was a small variation in the projectile mass, hence all results were scaled relative to $L+1 / 3 d=m /\left(r_{p} A\right)$ where $r_{p}$ is the projectile density taken equal to $7,830 \mathrm{~kg} / \mathrm{m}^{3}$. In the second set of experiments [5], $r_{p}$ was taken equal to $8,000 \mathrm{~kg} / \mathrm{m}^{3}$ and the projectiles had various lengths and diameters; this might have been the reason for the wider spread of the results. The value $p_{0}=1.82 \mathrm{GPa}$ was obtained by fitting the analytical results obtained from eqn (34) to the lower experimental values as shown in Fig. 4. It is seen in that figure that the predictions based on eqn (34) deviate considerably from the experimental results for higher impact velocities. This can be attributed to the additional target resistance due to cavitation.

The analytical integration of eqn (1) with $F$ given by eqn (24), $\lambda$ by eqn (31) for $\dot{x}>\dot{x}_{c}$ and $\lambda=1$ for $\dot{x} \leq \dot{x}_{c}$ gives

$$
x_{\max }=\frac{2 m \dot{x}_{c}^{2}}{A p_{0}}\left(\ln \frac{1+\alpha_{0}^{2}}{2}+\frac{1}{1+\alpha_{0}^{2}}-\frac{1}{4}\right)
$$

where $\alpha_{0}=\dot{x}_{0} / \dot{x}_{c} \geq 1$. Adopting a cavitation velocity value of around $450 \mathrm{~m} / \mathrm{s}$, eqn (35) provides the second set of analytical results shown in Fig. 4, which are in good agreement with the experimental data. The corresponding value of $K$, obtained from eqn (12), is around 3.31, which is close to the one identified earlier based on comparison with the numerical output of Batra and Wright [9]. 


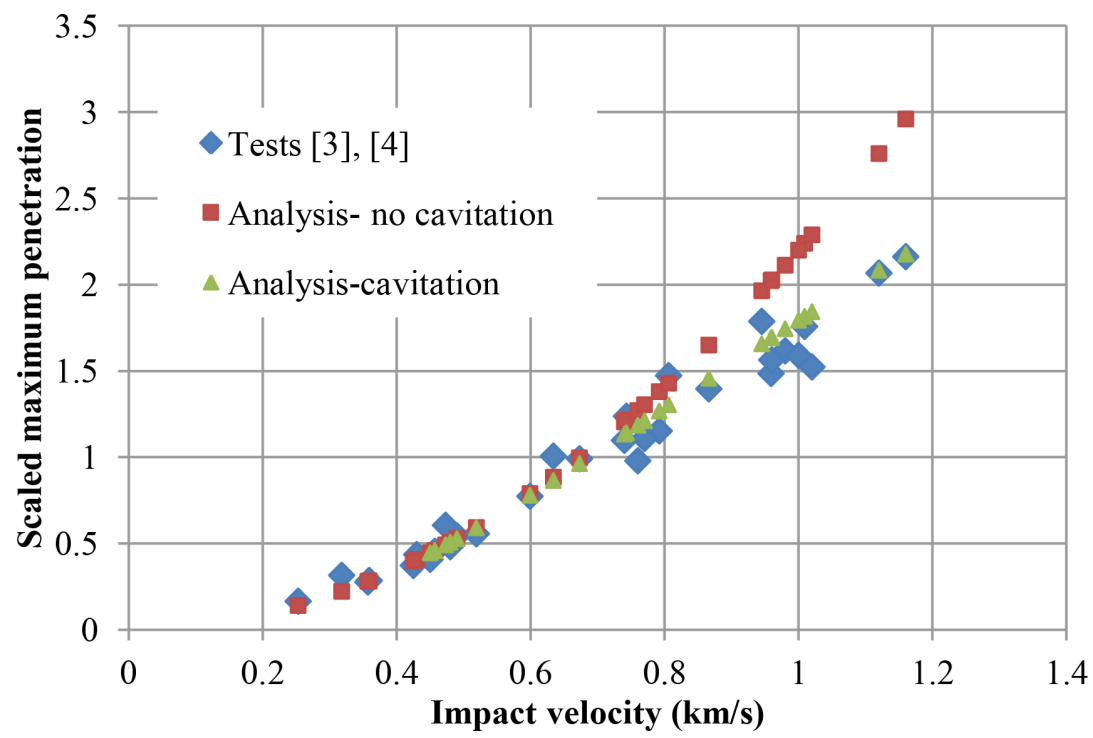

Figure 4: Experimental data and analytical predictions for hemispherical head projectiles against aluminium targets.

The same strategy can be applied to obtain the model parameters for ogival heads. Experimental results for $n=3$ are shown in Fig. 5 [4]. As pointed out earlier, it is reasonable to assume $k=1$ for this value of $n$, thus $\lambda$ is given by eqn (27) in the case of cavitation. Maximum penetration for $\dot{x}_{0} \leq \dot{x}_{c}$ is again given by eqn (34). The analysis for $\dot{x}_{0}>\dot{x}_{c}$ is now more

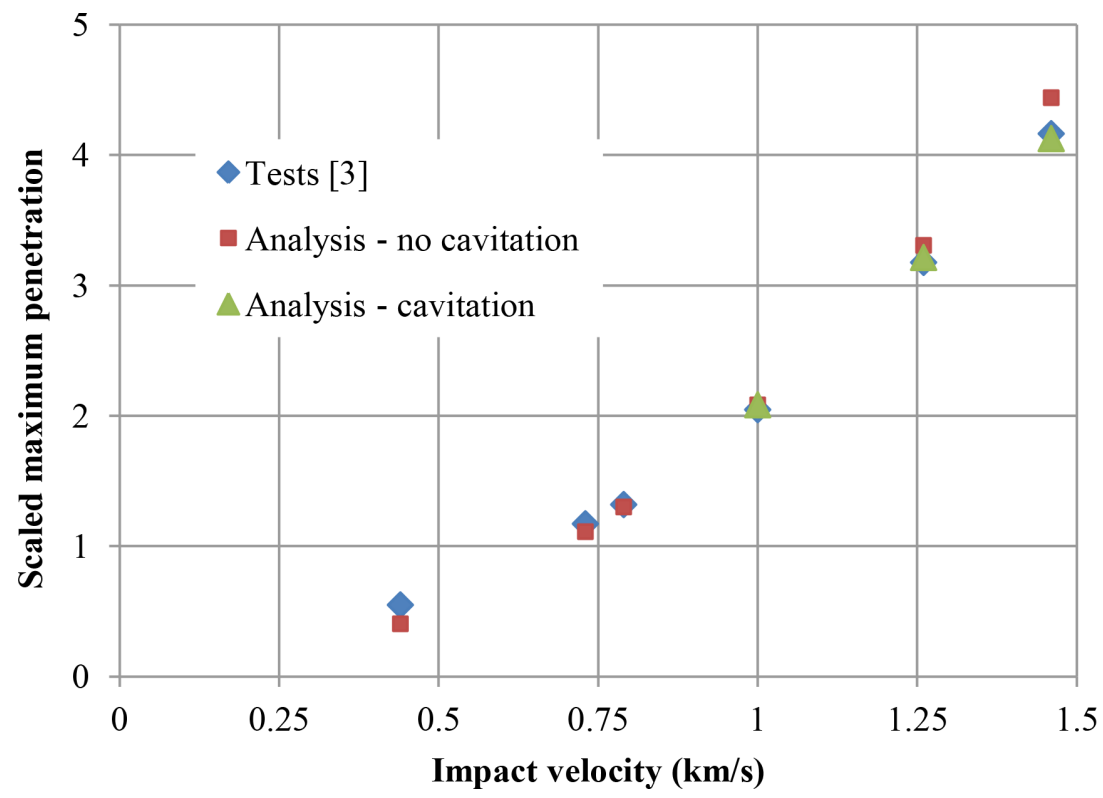

Figure 5: Experimental data and analytical predictions for ogival head projectiles against aluminium targets. 
complex since the equation of motion cannot be integrated explicitly. Thus, numerical quadrature was used, implemented through a short FORTRAN program.

Fitting the analytical predictions of maximum penetration to the corresponding experimental data as shown in Fig. 5 provided estimates for $p_{0}, \dot{x}_{c}$ and $K$ equal to $1.87 \mathrm{GPa}, 900 \mathrm{~m} / \mathrm{s}$ and 5.14, respectively. It is worth noting that, for the same target material, the hardness parameter is very close to that obtained with the hemispherical head, while parameter $K$, controlling the hydrodynamic contribution, increases significantly with $n$.

The model parameters are expected to depend also on the mechanical properties of the target material. This was confirmed by fitting the analytical predictions to another set of experimental data obtained using again ogive-headed projectiles with $n=3$ [6]. The target material in these experiments was an aluminium alloy with $\sigma_{Y}=448 \mathrm{MPa}$ and $\sigma_{f}(0.75)=675 \mathrm{MPa}$ in contrast to that of the earlier experiments for which $\sigma_{Y}=276 \mathrm{MPa}$ and $\sigma_{f}(1.00)=375 \mathrm{MPa}$. The parameter values estimated from these data were $p_{0}=2.25 \mathrm{GPa}$ and $K=7.9$, leading to $\dot{x}_{c}=790 \mathrm{~m} / \mathrm{s}$. These values reflect the higher resistance to penetration expected from harder materials.

It is noted in both Figs 4 and 5 that, for low impact velocities, the predicted penetration is less than the actual one. This may be due to ignoring the effect of the initial penetration phase during which both contact area and material constraint is less than assumed thereby actually providing less resistance to penetration. Evaluation of the extent of penetration during this phase requires numerical time-step integration since the resistive force depends on both $x$ and $\dot{x}$ according to eqn (15).

\section{CONCLUDING REMARKS}

Hill's model is an elegant mathematical representation of the penetration phenomenon but it has not been given sufficient attention in the ballistics literature. It was shown here how its scope could be expanded by enriching it with an additional parameter and linking its predictions with experimental evidence.

The importance of the new form for the drag coefficient proposed here, which depends on the additional parameter $k$, lies in its allowing the application of the model to make a smooth transition from projectiles with blunt hemispherical heads to those with slender, pointed, ogival or conical heads.

For this model to be useful in practice, it should be fully and reliably characterised under a wide range of conditions. It is therefore necessary to be tested against the relevant output from every available numerical and experimental work and this requires considerable effort. Additional experimental data for projectiles with ogive and hemispherical heads, such as those reported by Piekutowski et al. [7] and Forrestal and Piekutowski [8] as well as numerical simulations, such as that by Chen [12], can be found in the literature and used to confirm the validity of the model.

It would be desirable to establish empirical relations between the various model parameters and the kinematic, geometric and material characteristics of the penetration event. A possible relation between the exponent $k$ and the ogival parameter $n$ was suggested in the previous section but remains to be verified. The drag factor $K$ depends on both $n$ and target material strength; the results of the previous section suggest that $K$ decreases as $n$ decreases and increases as $E / \sigma_{Y}$ decreases. The latter conclusion is contrary to the trend suggested by eqn (11) but is consistent with Hill's estimate of $K(n=1.5)=2.8$ for mild steel, which has a higher $E / \sigma_{Y}$ ratio than an aluminium alloy.

The hardness term $p_{0}$ can be estimated from eqn (9) using one of the expressions for the constraint factor $a$ suggested in the literature $[4,11]$. Uncertainty however remains regarding 
the appropriate value of the flow stress due to its strain-rate dependence. Malvern's model [13] expresses this dependence and has been fitted to steel and aluminium alloy data [14], it is however difficult to assess a relevant value of the strain rate, which varies in both space and time. This can be resolved by resorting to numerical work such as that by Lin and Batra [15].

\section{REFERENCES}

[1] Syngellakis, S., Simple models for penetration of thick targets by rigid projectiles. WIT Transactions on the Built Environment, 126, pp. 63-74, 2012. https://doi.org/10.2495/SU120061

[2] Backman, M.E. \& Goldsmith, W., The mechanics of penetration of projectiles into targets. International Journal of Engineering Science, 16(1), pp. 1-99, 1978. https://doi.org/10.1016/0020-7225(78)90002-2

[3] Hill, R., Cavitation and the influence of headshape in attack of thick targets by nondeforming projectiles. Journal of Mechanics and Physics of Solids, 28(5-6), pp. 249263, 1980. https://doi.org/10.1016/0022-5096(80)90019-8

[4] Forrestal, M.J., Okajima, K. \& Luk, V.K., Penetration of 6061-T651 aluminium targets with rigid long rods. Journal of Applied Mechanics, Transactions of the ASME, 55(4), pp. 755-760, 1988.

[5] Forrestal, M.J., Brar, N.S. \& Luk, V.K., Penetration of strain-hardening targets with rigid spherical-nose rods. Journal of Applied Mechanics, Transactions of the ASME, 58, pp. 7-10, 1991.

https://doi.org/10.1115/1.2897183

[6] Forrestal, M.J., Luk, V.K., Rosenberg, Z. \& Brar, N.S., Penetration of 7075-T651 aluminum targets with ogival-nose rods. International Journal of Solids and Structures, 29(14/15), pp. 1729-1736, 1992. https://doi.org/10.1016/0020-7683(92)90166-Q

[7] Piekutowski, A.J., Forrestal, M.J., Poormon, K.L. \& Warren, T.L., Penetration of 6061T6511 aluminum targets by ogive-nose steel projectiles with striking velocities between 0.5 and 3.0 km/s. International Journal of Impact Engineering, 23(1), pp. 723-734, 1999. https://doi.org/10.1016/S0734-743X(99)00117-7

[8] Forrestal, M.J. \& Piekutowski, A.J., Penetration experiments with 6061-T6511 aluminum targets and spherical-nose steel projectiles at striking velocities between 0.5 and $3.0 \mathrm{~km} / \mathrm{s}$. International Journal of Impact Engineering, 24(1), pp. 57-67, 2000. https://doi.org/10.1016/S0734-743X(99)00033-0

[9] Batra, R.C. \& Wright, T.W., Steady state penetration of rigid perfectly plastic targets. International Journal of Engineering Science, 24(1), pp. 41-54, 1986. https://doi.org/10.1016/0020-7225(86)90147-3

[10] Jayachandran, R. \& Batra, R.C., Steady state penetration of elastic plastic targets. Acta Mechanica, 92, pp. 9-27, 1992. https://doi.org/10.1007/BF01174164

[11] Wright, T.W. \& Frank, K., Approaches to penetration problems. Impact: Effects of Transient Loadings, eds W.J. Amman, W.K. Liu, J.S. Studer \& T. Zimmermann, Balkema: Rotterdam, pp. 85-103, 1988.

[12] Chen, E.P., Numerical simulation of penetration of aluminium targets by sphericalnose steel rods. Theoretical and Applied Fracture Mechanics, 22(2), pp. 159-164, 1995. https://doi.org/10.1016/0167-8442(94)00056-7 
[13] Malvern, L.E., The propagation of longitudinal waves of plastic deformation in a bar of material exhibiting a strain rate effect. Journal of Applied Mechanics, 18, pp. 203-208, 1951.

[14] Symonds, P.S., Viscoplastic behavior in response of structures to dynamic loading. Behaviour of materials under dynamic loading, ed. N.J. Huffington, ASME: New York, pp. 106-124, 1965.

[15] Lin, P.R. \& Batra, R.C., Histories of the stress, strain-rate, temperature and spin in steady state penetration problems. International Journal of Engineering Science, 27(10), pp. 1155-1165, 1989.

https://doi.org/10.1016/0020-7225(89)90095-5 